Accepted for Publication in The Astrophysical Journal

\title{
X-Ray and Gamma-Ray Polarization in Leptonic and Hadronic Jet Models of Blazars
}

\author{
H. Zhang ${ }^{1}$ and M. Böttcher ${ }^{2,1}$
}

\begin{abstract}
We present a theoretical analysis of the expected X-ray and $\gamma$-ray polarization signatures resulting from synchrotron self-Compton emission in leptonic models, compared to the polarization signatures from proton synchrotron and cascade synchrotron emission in hadronic models for blazars. Source parameters resulting from detailed spectral-energy-distribution modeling are used to calculate photonenergy-dependent upper limits on the degree of polarization, assuming a perfectly organized, mono-directional magnetic field. In low-synchrotron-peaked blazars, hadronic models exhibit substantially higher maximum degrees of X-ray and gamma-ray polarization than leptonic models, which may be within reach for existing X-ray and $\gamma$-ray polarimeters. In high-synchrotron-peaked blazars (with electron-synchrotron-dominated X-ray emission), leptonic and hadronic models predict the same degree of X-ray polarization, but substantially higher maximum $\gamma$-ray polarization in hadronic models than leptonic ones. These predictions are particularly relevant in view of the new generation of balloon-borne X-ray polarimeters (and possibly GEMS, if revived), and the ability of Fermi-LAT to measure $\gamma$-ray polarization at $<200 \mathrm{MeV}$. We suggest observational strategies combining optical, X-ray, $\gamma$-ray polarimetry to determine the degree of ordering of the magnetic field and to distinguish between leptonic and hadronic high-energy emission.
\end{abstract}

Subject headings: galaxies: active — galaxies: jets — gamma-rays: galaxies radiation mechanisms: non-thermal — relativistic processes

\footnotetext{
${ }^{1}$ Astrophysical Institute, Department of Physics and Astronomy, Ohio University, Athens, OH 45701, USA

${ }^{2}$ Centre for Space Research, North-West University, Potchefstroom, 2520, South Africa
} 


\section{Introduction}

While measurements of synchrotron polarization of the radio and optical emission from relativistic jet sources (blazars, radio galaxies, gamma-ray bursts) have become a standard way of assessing the degree of order and direction of magnetic fields, the polarization of high-energy (X-ray and $\gamma$-ray) emission has so far remained largely unexplored. However, there are several projects underway to develop balloon- and satellite-borne X-ray polarimeters: Current balloon-borne X-ray polarimetry experiments include PoGoLite $(2-100 \mathrm{keV}$; Pearce et al. 2012), X-Calibur (20 - $80 \mathrm{keV;} \mathrm{Beilicke} \mathrm{et} \mathrm{al.} \mathrm{2012),} \mathrm{and} \mathrm{POLAR} \mathrm{(50} \mathrm{-} 500 \mathrm{keV;}$ Orsi 2011). As satellite-borne instruments, SPI and IBIS on board the INTEGRAL satellite have already been used successfully to constrain the hard X-ray / soft $\gamma$-ray polarization from gamma-ray bursts (Dean et al. 2008; Forot et al. 2008), and design studies for the upcoming ASTRO-H mission suggest that it may also be able to detect polarization in the 50 - $200 \mathrm{keV}$ energy band (Tajima et al. 2010). Estimates of the minimum detectable degree of polarization for most of these instruments range around $10 \%$ for moderately bright X-ray sources. Unfortunately, the Gravity and Extreme Magnetism SMEX (GEMS; 2 - $10 \mathrm{keV}$; Swank et al. 2010) was recently cancelled by NASA, in spite of being rather far along in its developing phase. It has also been suggested that the Large Area Telescope (LAT) on-board the Fermi Gamma-Ray Space Telescope may be able to detect $\gamma$-ray polarization in the energy range $\sim 30-200 \mathrm{MeV}$ when considering pair-conversion events occurring in the Silicon layers of the detector, by taking advantage of the polarization-dependent direction of motion of the electron-positron pairs produced in the $\gamma$-ray - pair conversion process (Bühler et al. 2010). For bright $\gamma$-ray sources, degrees of polarization down to $\sim 10 \%$ may be detectable.

On the theory side, the general formalism for calculating X-ray and $\gamma$-ray polarization has been well developed. It is well-known that linear polarization arises from synchrotron radiation of relativistic charged particles in ordered magnetic fields, while Compton scattering off relativistic electrons will reduce the degree of polarization of the target photon field, without entirely destroying it. Compton scattering of unpolarized target photon fields by isotropic distributions of electrons (and positrons) will always result in un-polarized Compton emission. The well-known formalism for calculating synchrotron polarization can be found, e.g., in the text book by Rybicki \& Lightman (1985). A formalism for evaluating the polarization of Compton scattered radiation in the Thomson regime was developed by Bonometto et al. (1970) and applied specifically to synchrotron self-Compton (SSC) emission by Bonometto \& Saggion (1973). More recently, Krawczynski (2012) provided a general Monte-Carlo based framework for the evaluation of polarization signatures in relativistic environments in both Thomson and Klein-Nishina regimes, verifying that the expressions of Bonometto et al. (1970) and Bonometto \& Saggion (1973) are valid in the Thomson regime. 
However, while the general framework for the evaluation of polarization signatures from different radiation mechanisms exists, it has so far not been applied to realistic representations of the high-energy emission from relativistic jets of active galactic nuclei (AGN), which are the most numerous source class in the Fermi-LAT energy range. The $\gamma$-ray brightest AGN detected by Fermi-LAT are blazars, i.e., radio-loud (jet-dominated) AGN in which the jet points at a small angle with respect to our line of sight. Example results for the synchrotron and SSC polarization in blazar jets have been presented by Poutanen (1994), indicating that substantial $(>30 \%)$ polarization may result from these radiation mechanisms in the case of perfectly ordered magnetic fields. However, his calculations were restricted to pure power-law electron spectra and only a very specific choice of parameters, with no direct connection to the observed spectral energy distributions (SEDs) of blazars. The SEDs of blazars are dominated by non-thermal emission across the entire electromagnetic spectrum, with two broad components. Depending on the peak frequency of the low-frequency component (generally agreed to be synchrotron radiation from relativistic electrons), they are sub-divided between Low-Synchrotron-Peaked (LSP) blazars (consisting of flat-spectrum radio quasars [FSRQs] and low-frequency peaked BL Lac objects [LBLs]), Intermediate-Synchrotron-Peaked (ISP) blazars (generally intermediate BL Lac objects [IBLs]), and High-Synchrotron-Peaked (HSP) blazars (which are exclusively high-frequency-peaked BL Lac objects [HBLs]).

The mechanism producing the high-energy (X-ray through $\gamma$-ray) emission in blazars is still under debate. Both leptonic and hadronic models are currently still viable and are generally able to produce acceptable fits to the SEDs of most blazars (for a review of blazar emission models see, e.g., Böttcher 2010). Additional information, such as variability or polarization, is therefore needed to distinguish between leptonic and hadronic emission scenarios.

In this paper, we evaluate the expected X-ray and $\gamma$-ray polarization signatures in both leptonic and hadronic emission models for blazars, considering all sub-classes (LSP, ISP, and HSP) of blazars. In \$2, we briefly describe the formalism used to evaluate synchrotron and SSC polarization in this work, and the leptonic and hadronic blazar emission models considered. In 93 , we present our results comparing the frequency-dependent polarization signatures of leptonic and hadronic blazar models for blazars of all sub-classes. These results are used in $\$ 4$ to develop observational diagnostics based on optical, X-ray and $\gamma$-ray polarimetry to confidently distinguish between leptonic and hadronic emission models. We summarize in $\$ 5$, 


\section{Calculation of Synchrotron and Synchrotron-Self-Compton Polarization}

In this section, we will describe the formalism used in this paper to evaluate the degree of polarization from synchrotron and Compton scattering, and the specific leptonic and hadronic blazar models used to provide input parameters for the polarization calculations. All results shown here are based on the assumption of a perfectly ordered, mono-directional magnetic field, oriented perpendicular to the line of sight. This configuration produces the maximum possible degree of polarization both for synchrotron and SSC emission. Thus, our results represent upper limits to the actually expected polarization from emission regions with partially dis-ordered magnetic fields. As we will elaborate in Section 4, the observed degree of polarization in frequency ranges in the SED that can be confidently ascribed to synchrotron radiation can then be used to re-normalize our results to find the actually expected degree of X-ray and $\gamma$-ray polarization.

All particle distributions, on which we base our calculations, are assumed to be isotropic in the co-moving frame of the emission region, as is routinely done in blazar emission models. This is justified by very efficient pitch-angle scattering on small-scale magnetic turbulence (with small amplitudes $\delta B / B_{0} \ll 1$, where $B_{0}$ is the ordered background magnetic field) which is expected to be present in the highly relativistic blazar environment. For a more detailed discussion of this aspect, see, e.g., Böttcher et al. (1997).

In general terms, we will evaluate the radiation powers $P_{\|}$and $P_{\perp}$ of radiation with electric-field vectors parallel and perpendicular, respectively, to the projection of the magnetic field onto the plane of the sky (which is identical to the actual magnetic field in the configuration chosen for our calculations). The degree of polarization $\Pi$ is then evaluated as

$$
\Pi(\omega)=\frac{P_{\perp}(\omega)-P_{\|}(\omega)}{P_{\perp}(\omega)+P_{\|}(\omega)}
$$

\subsection{Synchrotron and Synchrotron-Self-Compton Polarization}

For the case of synchrotron emission, $P_{\|}(\omega)$ and $P_{\perp}(\omega)$ are evaluated by integrating the single-particle powers $P_{\|}(\omega, \gamma)$ and $P_{\perp}(\omega, \gamma)$ from Eqs. (6.32a,b) in Rybicki \& Lightman (1985) over the electron spectrum $N_{e}(\gamma)$, e.g., $P_{\|, \perp}(\omega)=\int_{1}^{\infty} P_{\|, \perp}(\omega, \gamma) N_{e}(\gamma) d \gamma$. This procedure is valid for arbitrary particle spectra (not only the most commonly considered power-law case) and is followed for synchrotron emission from all relevant species, i.e., electrons in both leptonic and hadronic models as well as protons and electron-positron pairs from cascades in hadronic models. Our calculations properly account for the mass difference in the case of 
proton synchrotron (see, e.g. Aharonian 2000). It is well known that a power-law spectrum of electrons $N_{e}(\gamma) \propto \gamma^{-p}$ (producing a power-law synchrotron radiation spectrum $F_{\nu} \propto \nu^{-\alpha}$ with $\alpha=(p-1) / 2$ ) will result in a polarization degree of (Rybicki \& Lightman 1985)

$$
\Pi_{\text {powerlaw }}=\frac{p+1}{p+7 / 3}=\frac{\alpha+1}{\alpha+5 / 4}
$$

For Compton scattering, we follow the formalism of Bonometto \& Saggion (1973). We only need to calculate the polarization for SSC emission since X-ray and $\gamma$-ray emission from Compton scatering of radiation fields external to the $\gamma$-ray emission region $(\mathrm{EC}=$ External Compton) is expected to be unpolarized, and can simply be added as a radiation component with $P_{\|}^{\mathrm{EC}}(\omega)=P_{\perp}^{\mathrm{EC}}(\omega)$.

Since the expressions for SSC polarization given by Bonometto \& Saggion (1973) are only valid in the Thomson regime, we must first confirm their validity in the considered energy range of $\sim 0.1 \mathrm{keV}-500 \mathrm{MeV}$, for which we will show polarization results in Section 3 in order to encompass the energy ranges covered by X-ray polarimeters and Fermi-LAT's polarization capabilities. Here and throughout the paper, we define a dimensionless photon energy $\epsilon \equiv h \nu /\left(m_{e} c^{2}\right)$. Doppler boosting from the co-moving to the observer's frame is described by the Doppler factor $\delta \sim 10$ for typical blazar sources. In the Thomson regime, the observed Compton-scattered photon energy $\epsilon_{C}^{\text {obs }}$ is given by $\epsilon_{C}^{\text {obs }}=\gamma^{2} \delta \epsilon_{s}$, where $\epsilon_{s}$ is dimensionless target photon energy in the co-moving frame of the $\gamma$-ray emission region. For an observed photon energy of $E_{C}^{\mathrm{obs}}=500 \mathrm{MeV}, \epsilon_{C}^{\mathrm{obs}} \sim 1000$. In the SSC process, the target synchrotron photons have a typical photon energy around $\hbar \omega \sim \mathrm{eV}$, so that $\epsilon_{s} \sim 10^{-6}$. Hence, $\gamma \epsilon_{s}=\sqrt{\epsilon_{C}^{\mathrm{obs}} \epsilon_{s} \delta^{-1}} \sim 0.01 \ll 1$. We can therefore safely work in the Thomson regime, for which Krawczvnski (2012) has verified with detailed Monte-Carlo simulations that the expressions of Bonometto \& Saggion (1973) provide an excellent description of the polarization signatures.

Let $\mathbf{k}, \epsilon$ and $\mathbf{k}^{\prime}, \epsilon^{\prime}$ be the momentum and the frequency of the photons before and after scattering, with unit vectors $\mathbf{v}_{\mathbf{k}}=\mathbf{k} /|\mathbf{k}|$ and $\mathbf{v}_{\mathbf{k}^{\prime}}=\mathbf{k}^{\prime} /\left|\mathbf{k}^{\prime}\right|$. In the Thomson regime, the power emitted at frequency $\epsilon^{\prime}$ resulting from scattering of photons whose original polarization direction is e (taken as being perpendicular to the magnetic field for synchrotron target photons), scattered into two orthogonal polarization directions ( $\mathbf{e}^{\prime}$ parallel or perpendicular to the projection of the magnetic field onto the plane orthogonal to $\left.\mathbf{k}^{\prime}\right), P_{\|}^{\mathrm{SSC}}$ and $P_{\perp}^{\mathrm{SSC}}$, are given by (Bonometto \& Saggion 1973, converted to c.g.s units)

$$
P_{\|}^{\mathrm{SSC}}\left(\epsilon^{\prime}\right)=\pi\left(\frac{e^{2}}{4 \pi}\right)^{2} \frac{c}{m_{e} c^{2}} \epsilon^{\prime} \int \frac{d \epsilon}{\epsilon} d \Omega_{k} E_{\min } n(\epsilon) q(\vartheta) \cdot\left(Z_{\|}\left(\Sigma_{1}+\Sigma_{2}\right)+\Sigma_{2}\right)
$$




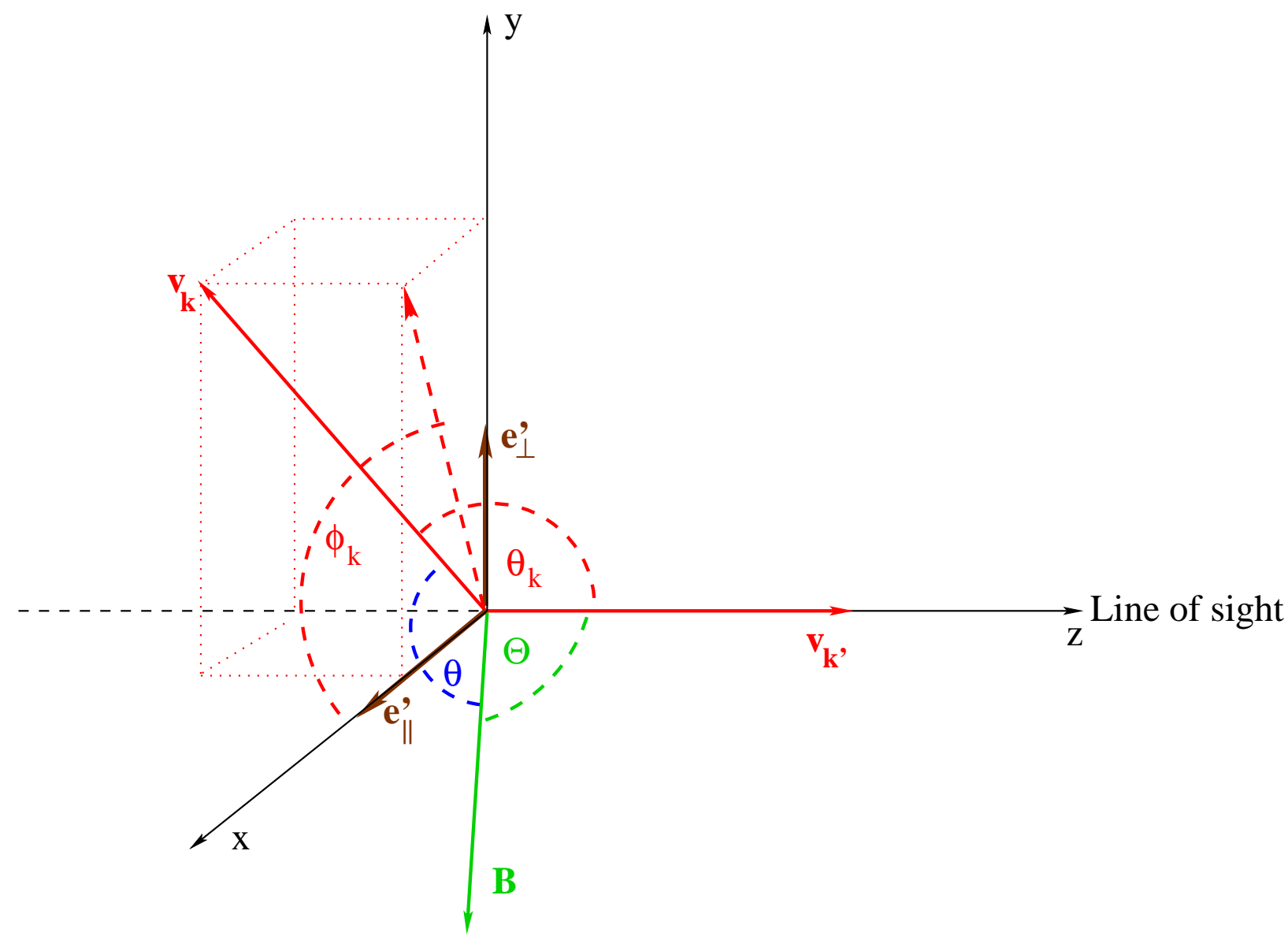

Fig. 1. - Sketch of the scattering geometry to illustrate the definition of angles. The coordinate system is chosen such that the magnetic field $\mathbf{B}$ lies in the $(\mathrm{x}-\mathrm{z})$ plane. See the electronic edition of the Journal for a color version of this figure.

$$
P_{\perp}^{\mathrm{SSC}}\left(\epsilon^{\prime}\right)=\pi\left(\frac{e^{2}}{4 \pi}\right)^{2} \frac{c}{m_{e} c^{2}} \epsilon^{\prime} \int \frac{d \epsilon}{\epsilon} d \Omega_{k} E_{\min } n(\epsilon) q(\vartheta) \cdot\left(Z_{\perp}\left(\Sigma_{1}+\Sigma_{2}\right)+\Sigma_{2}\right)
$$

where

$$
E_{\text {min }}=\sqrt{\frac{\epsilon^{\prime}}{2 \epsilon\left(1-\cos \theta_{k}\right)}}
$$

is the minimum electron energy required for scattering of a photon from $\epsilon$ to $\epsilon^{\prime}$,

$$
Z_{\mathbf{e}^{\prime}}=\left(\mathbf{e} \cdot \mathbf{e}^{\prime}+\frac{\left(\mathbf{v}_{\mathbf{k}} \cdot \mathbf{e}^{\prime}\right)\left(\mathbf{v}_{\mathbf{k}^{\prime}} \cdot \mathbf{e}\right)}{1-\cos \theta_{k}}\right)^{2}
$$

and we have defined the solid angle of the direction of the photon before scattering, $\mathbf{k}$, 
as

$$
d \Omega_{\mathbf{k}}=d \cos \theta_{k} d \varphi_{k}
$$

$\vartheta$ is defined as the angle between the magnetic field and $\mathbf{k}$, which can be related to the angle $\Theta$ between the magnetic field and the line of sight $\left(\mathbf{k}^{\prime}\right)$ through

$$
\cos \vartheta=\cos \Theta \cos \theta_{k}+\sin \Theta \sin \theta_{k} \cos \varphi_{k}
$$

See Figure 1 for an illustration of the angle definitions.

The synchrotron photon distribution $n(\mathbf{k})$ has been separated into an energy spectrum and an angle-dependent function,

$$
n(\mathbf{k})=\frac{n(\epsilon) q(\vartheta)}{\epsilon^{2}}
$$

where the angle-dependence is chosen as $q(\vartheta) \propto \sin ^{\frac{p+1}{2}} \vartheta$ with $p$ being the local spectral index of the underlying electron spectrum, and the synchrotron spectrum $n(\epsilon)$ is calculated selfconsistently, using the full expressions of Rybicki \& Lightman (1985) for the given electron spectrum.

$$
\begin{aligned}
& \Sigma_{1}=\int_{\beta_{1}}^{\beta_{2}} d x m(E)\left(x^{2}-x^{-2}+2\right) \\
& \Sigma_{2}=\int_{\beta_{1}}^{\beta_{2}} d x m(E) \frac{\left(1-x^{2}\right)^{2}}{x^{2}}
\end{aligned}
$$

where $E$ is electron energy and

$$
\begin{aligned}
m(E) & =\frac{N_{e}(E)}{E^{2}} \\
x & =\frac{E_{\text {min }}}{E} \\
\beta_{1} & =\left\{\begin{array}{cc}
1 & E_{\text {min }}>E_{2} \\
\frac{E_{\text {min }}}{E_{2}} & E_{\text {min }}<E_{2}
\end{array}\right. \\
\beta_{2} & =\left\{\begin{array}{cc}
1 & E_{\text {min }}>E_{1} \\
\frac{E_{\min }}{E_{1}} & E_{\text {min }}<E_{1}
\end{array}\right.
\end{aligned}
$$

We have developed efficient codes to evaluate the synchrotron and SSC polarization for arbitrary, isotropic electron spectra in the case of perfectly ordered magnetic fields. Our 
code calculates the radiation powers $P_{\perp}(\omega)$ and $P_{\|}(\omega)$ from all relevant radiation mechanisms separately and then evaluates the total polarization degree as a function of photon frequency according to Eq. (1).

\subsection{Blazar Models}

We apply the polarization code described above to both leptonic and hadronic, stationary single-zone models of Fermi-detected blazars, as described in detail in Böttcher et al. (2013). The blazar models have been used to produce SED fits to a number of blazars of all sub-classes. The particle distributions and other model parameters (in particular, the magnetic field B) required by the models to reproduce an individual blazar's SED are then used to evaluate the photon-energy-dependent degree of polarization with the code described here.

In the leptonic blazar model, the high energy component of the SED has contributions from synchrotron-self-Compton (SSC) and external Compton (EC) radiation, whose seed photons are from direct accretion disk emission, accretion disk emission reprocessed by the Broad Line Region (BLR) and an isotropic external radiation field. Since the EC high-energy emission is expected to be un-polarized, we only need to evaluate the synchrotron and SSC polarization, and we obtain the total polarization degree in the leptonic model as

$$
\Pi(\omega)=\frac{P_{\perp}^{\mathrm{sy}+\mathrm{SSC}}(\omega)-P_{\|}^{\mathrm{sy}+\mathrm{SSC}}(\omega)}{P_{\perp}^{\mathrm{sy}+\mathrm{SSC}}(\omega)+P_{\|}^{\mathrm{sy}+\mathrm{SSC}}(\omega)} \cdot \frac{P_{\mathrm{sy}}(\omega)+P_{\mathrm{SSC}}(\omega)}{P_{\text {Total }}(\omega)} .
$$

In the hadronic model, the high-energy emission consists primarily of contributions from proton synchrotron emission and synchrotron emission from secondary pairs produced in cascade processes initiated by photo-pion production. At lower energies, especially in the X-ray range, the SSC emission of the primary electrons (primarily responsible for the low-frequency synchrotron peak) may also make a non-negligible contribution. This model only evaluates the spectra of the final decay products of the photo-pion production, neglecting the emission from intermediate decay products such as muons. Thus, the polarization is calculated taking into account contributions from synchrotron emissions of primary electrons and protons and from secondary electron-positron pairs, as well as SSC from primary electrons. We point out that, even if muon and pion synchrotron emission makes a substantial contribution to the $\gamma$-ray emission, such a component would be expected to be equally highly polarized as the proton and pair synchrotron emission considered in the model. Thus, a variant of the hadronic model that produces the same SED, but including non-negligible contributions from muon and pion synchrotron emission, is expected to yield very similar polarization sig- 
natures as calculated here. Even though the target (synchrotron) photon field for photopair and photopion production is polarized in the configuration considered here, our code neglects any potential dependence of the relevant $\mathrm{p} \gamma$ cross sections on the polarization of the target photon field, as such dependence is very poorly understood and due to the assumed isotropy of the proton distribution, its effects are expected to be negligible.

\section{Results and Discussion}

We have applied the method of calculating the frequency-dependent degree of polarization to a substantial number of Fermi-detected FSRQs, LBLs, IBLs, and HBLs. We have evaluated the degree of polarization between $0.1 \mathrm{keV}$ and $500 \mathrm{MeV}$, encompassing the energy range in which X-ray polarimeters and Fermi-LAT have realistic prospects of measuring high-energy polarization. We remind the reader that the results shown here are upper limits to the polarization, assuming a perfectly ordered magnetic field perpendicular to the line of sight (in the co-moving frame of the emission region).

$3 \mathrm{C} 279$

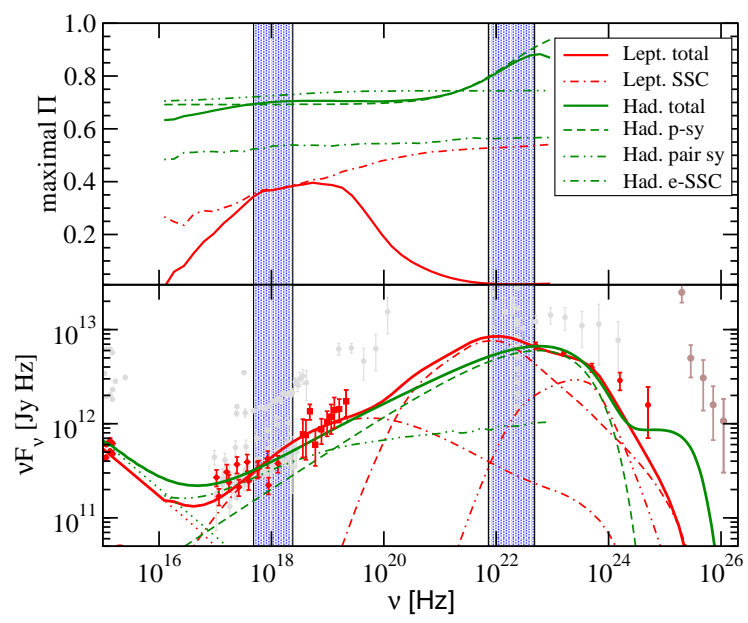

PKS 0528+134

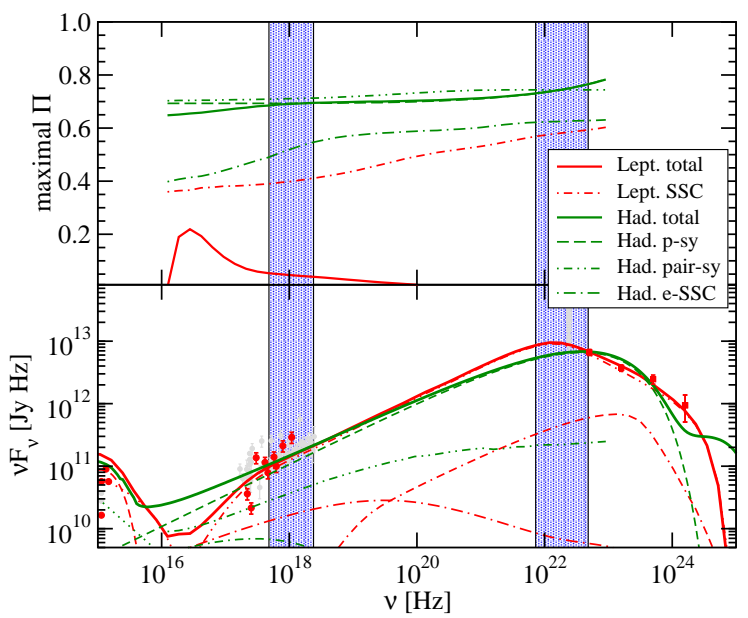

Fig. 2.- UV through $\gamma$-ray SEDs (lower panels) and maximum degree of polarization (upper panels) for the two FSRQs 3C279 (left) and PKS 0528+134 (right). Leptonic model fits are plotted in red, hadronic models in green. Different line styles indicate individual radiation components, as labeled in the legend. Shaded areas indicate the $2-10 \mathrm{keV}$ X-ray range (Xray polarimeters) and the $30-200 \mathrm{MeV}$ range, in which $\gamma$-ray emission may be measurable by Fermi-LAT. See the electronic edition of the Journal for a color version of this figure. 


\subsection{Low-Synchrotron-Peaked Blazars}

Figure 2 shows the results of SED fitting (from Böttcher et al. 2013, lower panels) and the photon-energy-dependent degree of polarization (top panels) throughout the X-ray and $\gamma$-ray regime, for two representative FSRQs. In the case of FSRQs, the synchrotron emission from electrons (i.e., primary electrons in the hadronic model) does generally not contribute appreciably in the X-ray (or higher-energy) range. In leptonic models, the highenergy emission is typically dominated by SSC throughout the X-ray regime, while at $\gamma$-ray energies, EC tends to dominate. In hadronic models, the high-energy emission of LSP blazars is typically well reproduced by models strongly dominated by proton synchrotron emission.

The first, obvious result to be seen in Fig. 2 is that leptonic models predict systematically lower degrees of polarization than (synchrotron-dominated) hadronic models. The SSC process reduces the polarization of the synchrotron seed photons to values typically not exceeding $\Pi_{\mathrm{SSC}} \lesssim 40 \%$, while the proton synchrotron emission may be polarized up to $\Pi_{\mathrm{SSC}} \sim 70-75 \%$ (in agreement with Eq. 2 for proton spectral indices $p \sim 2-3$ ), with the polarization gradually increasing due to the generally convex shape of the $\gamma$-ray SED (increasing $p$ implying increasing $\Pi$ ). Furthermore, due to the transition from SSC to EC from the X-ray to the $\gamma$-ray regime in leptonic models, the degree of polarization is expected to decrease rapidly with photon energy, and vanish in the Fermi-LAT energy range.

OJ 287

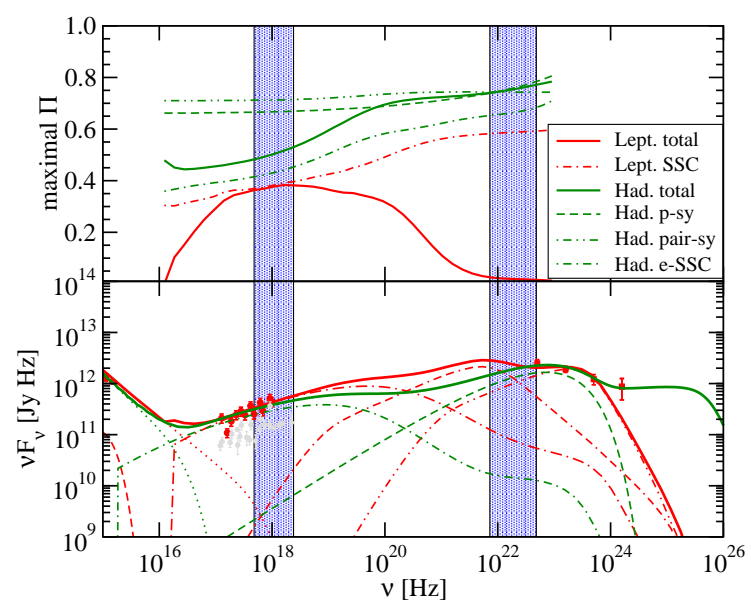

BL Lacertae

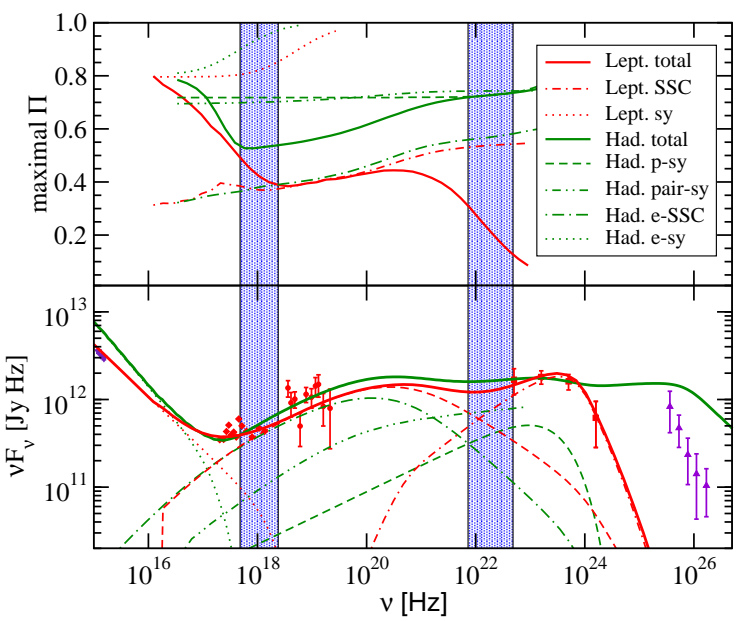

Fig. 3.- UV through $\gamma$-ray SEDs (lower panels) and maximum degree of polarization (upper panels) for the two LBLs OJ 287 (left) and BL Lacertae (right). Same symbol/color coding as in Fig. 2. See the electronic edition of the Journal for a color version of this figure.

Some LBLs exhibit very similar X-ray and $\gamma$-ray polarization signatures as those dis- 
cussed for FSRQs above. However, the hadronic fits of Böttcher et al. (2013) to several LBLs require their X-ray emission to be electron-SSC dominated, as in the leptonic-model case. Figure 3 shows two such examples. In that case, the degree of X-ray polarization predicted by hadronic models is substantially lower than in the FSRQ case and only slightly higher than predicted by leptonic models. In some cases (e.g., BL Lacertae, see Fig. 3, right), the X-ray emission also contains a non-negligible contribution from electron-synchrotron radiation, which may increase the expected maximum degree of polarization, especially in the leptonic model, and thereby further decrease the difference between the leptonic and hadronic model predictions. At $\gamma$-ray energies, the same drastic difference between the predicted degrees of polarization persists: Hadronic models predict up to $\sim 70-75 \%$ maximum $\gamma$-ray polarization, with substantially lower $\gamma$-ray polarization predicted by leptonic models.

3C66A

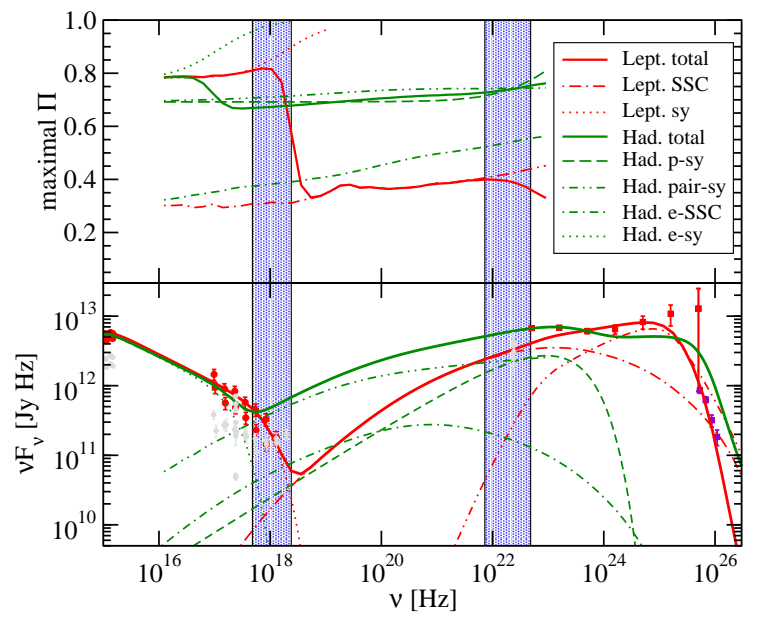

W Comae

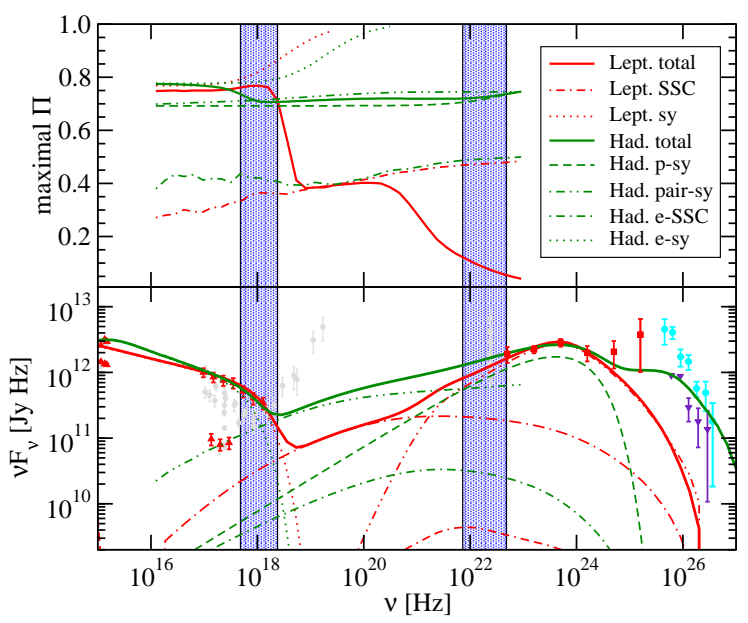

Fig. 4.- UV through $\gamma$-ray SEDs (lower panels) and maximum degree of polarization (upper panels) for the two IBLs 3C66A (left) and W Comae (right). Same symbol/color coding as in Fig. 2, See the electronic edition of the Journal for a color version of this figure.

\subsection{Intermediate-Synchrotron-Peaked Blazars}

Figure 4 shows the results of SED fitting (also from Böttcher et al. 2013) and frequencydependent high-energy polarization for two representative IBLs. In the case of IBLs, the $\mathrm{X}$-ray regime often covers the transition region from synchrotron (i.e., primary electronsynchrotron in hadronic models) emission to Compton emission in leptonic models and proton-induced emission in hadronic models. Therefore, at soft X-ray energies, both leptonic and hadronic models can exhibit very high degrees of polarization, dominated by the steep 
high-energy tail of the low-frequency synchrotron component. Leptonic models reproduce the hard X-ray through $\gamma$-ray emission typically with SSC dominated emission, although in the high-energy $\gamma$-ray regime $(E \gtrsim 100 \mathrm{MeV})$, an additional $\mathrm{EC}$ component is often required (e.g., Acciari et al. 2009; Abdo et al. 2011). Consequently, the degree of polarization is expected to decrease rapidly with energy to maximum values of typically $\sim 30 \%$ throughout the hard X-ray to soft $\gamma$-ray band, and may decrease even further if the HE $\gamma$-ray emission is EC dominated. Hadronic models often require contributions from proton-synchrotron, pair synchrotron, and primary-electron SSC emission. This SSC contribution slightly lowers the hard X-ray through soft $\gamma$-ray polarization compared to purely synchrotron-dominated emission (as in most LSPs), but still predicts substantially higher degrees of hard X-ray and $\gamma$-ray polarization compared to leptonic models.

RBS 0413

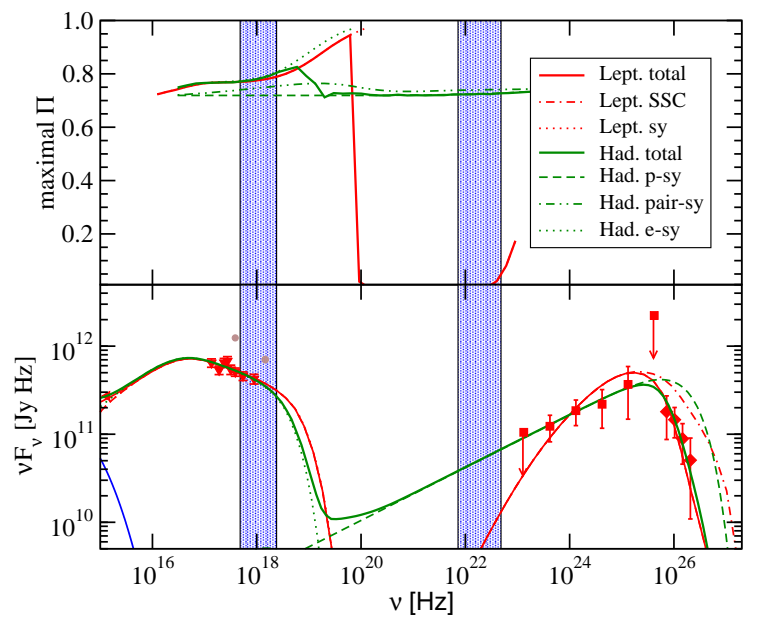

RX J0648.7+1516

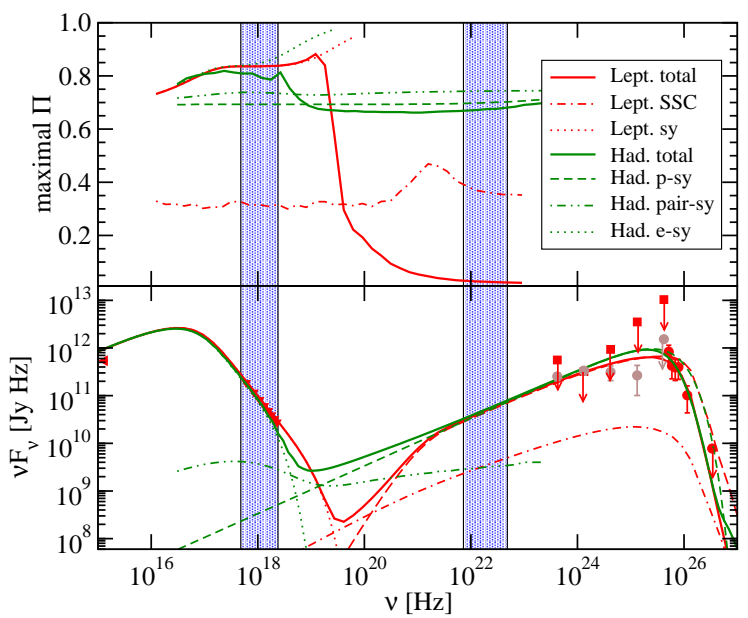

Fig. 5.- UV through $\gamma$-ray SEDs (lower panels) and maximum degree of polarization (upper panels) for the two HBLs RBS 0413 (left) and RX J0648.7+1516 (right). Same symbol/color coding as in Fig. 2. See the electronic edition of the Journal for a color version of this figure.

\subsection{High-Synchrotron-Peaked Blazars}

Two examples of SED fits and corresponding maximum polarization in the case of HBLs are shown in Figure 5. The data and SED fits for RBS 0413 are from Aliu et al. (2012); those for RX J0648.7+1516 are from Aliu et al. (2011). In HBLs, the X-ray emission (at least below a few $10 \mathrm{~s}$ of $\mathrm{keV}$ ) is typically strongly dominated by electron-synchrotron radiation, in both leptonic and hadronic models. Therefore, both models make essentially identical predictions of high maximum polarization throughout the X-ray regime. In leptonic models, 
the $\gamma$-ray emission of HBLs is often well represented by pure SSC emission, although a few cases also require additional (or even dominant) contributions from EC (see the case of RX J0648.7+1516 in the right panel of Fig. 5 for one such example; Aliu et al. 2011). Combined with the very low soft $\gamma$-ray fluxes predicted by such models, $\gamma$-ray polarization from HBLs in leptonic models is not expected to be detectable. In hadronic models, the $\gamma$-ray emission of HBLs is usually well represented by proton-synchrotron dominated scenarios, predicting a high level of maximum polarization.

\section{Exploiting High-Energy Polarization to Distinguish Leptonic from Hadronic Emission}

As pointed out, the calculations presented here assume a perfectly ordered magnetic field and therefore only represent upper limits to the possible degree of polarization actually observed. Therefore, in the case of a non-detection of X-ray and $\gamma$-ray polarization, it would seem difficult to establish whether this is simply due to an un-ordered magnetic field, or would actually favour a leptonic emission mechanism. However, this ambiguity may be lifted if the degree of polarization is determined in energy ranges of the SED that can be confidently ascribed to synchrotron emission.

In the case of FSRQs and LBLs, it is generally agreed that the near infrared (NIR) optical emission is dominated by synchrotron radiation (e.g., Ghisellini et al. 1998). Optical polarimetry will therefore allow one to determine the degree of order/disorder of the magnetic field in the emission region by comparing the measured $\Pi_{\text {syn }}$ with the theoretical maximum given by Eq. (2), since the spectral index $\alpha$ of the NIR - optical synchrotron spectrum is easily measured. Due to the $\lambda^{2}$ dependence of Faraday rotation, the effects of Faraday depolarization are negligible at optical wavelengths, so that the polarization measured here does, indeed, provide a realistic estimate of the degree of order of the magnetic field. Our results, as illustrated in Figures 2 and 3 may then be re-scaled by that factor to arrive at a realistic prediction of the expected degree of X-ray and $\gamma$-ray polarization and to determine whether the expected degree of polarization (especially in hadronic models) is within the capabilities of X-ray polarimeters and Fermi-LAT. If it is, X-ray polarimetry may be able to distinguish SSC from proton-synchrotron-dominated emission. In the Fermi-LAT regime, the difference between hadronic and leptonic model is expected to be even more obvious, and a positive detection of $\gamma$-ray polarization by Fermi-LAT would strongly favor a hadronic emission scenario.

Optical polarimetry of LSP blazars often reveals $\Pi_{\mathrm{sy}}$ (opt) $\sim 10-20 \%$, compared to $\Pi_{\mathrm{sy}}^{\max } \sim 75 \%$ for a typical spectral index of $p \sim 3$. Consequently, a similar degree of X-ray 
and $\gamma$-ray polarization of $\Pi_{\text {psy }} \sim 10-20 \%$ may be expected for quasars in the case of hadronic emission, which is at the borderline of the capabilities of existing polarimeters.

In the case of IBLs and HBLs, where the X-rays (in IBLs only the soft X-rays) are dominated by synchrotron emission, the degree of order/disorder of the magnetic field may be determined directly from X-ray polarimetry by compmaring the measured $\Pi_{\text {sy }}$ to the theoretical maximum from synchrotron polarization, as evaluated by our calculations. As in the case of FSRQs, the resulting re-scaling factor may be used to assess whether the expected $\gamma$-ray polarization is within the capabilities of Fermi-LAT to determine polarization, and if so, hadronic emission is expected to reveal itself through a high degree of polarizaiton.

We should point out here that HBLs typically exhibit very hard photon spectra in the Fermi-LAT range, with very low photon fluxes below $200 \mathrm{MeV}$. The detection of $\gamma$-ray polarization in HBLs therefore seems infeasible in the near future. It has been suggested that HBLs may be prime targets for X-ray polarimeters since they tend to be the brightest blazars in X-rays. However, unfortunately, leptonic and hadronic models agree on the interpretation of the X-ray emission of HBLs as due to electron synchrotron radiation, and therefore make identical predictions for the X-ray polarization. Thus, X-ray polarimetry of HBLs is not expected to aid in distinguishing leptonic from hadronic emission, given current $\gamma$-ray polarimetry capabilities. Such diagnostics would require substantially increased sensitivity and/or $\gamma$-ray polarimetry at energies much above $200 \mathrm{MeV}$.

\section{Summary and Conclusions}

We have presented calculations of the maximum achievable degree of X-ray and $\gamma$-ray polarization expected in leptonic and hadronic one-zone models for blazars. We generally find that hadronic models predict very high degrees of maximum polarization for all classes of blazars, since the entire SED is dominated by synchrotron processes. Even when accounting for the expected deviations from perfectly ordered magnetic fields, the predicted degree of polarization may be within the capabilities of current X-ray and $\gamma$-ray polarimeters. While in LSPs, the degree of polarization is expected to continually increase with increasing photon energy, it is expected to remain roughly constant throughout the X-ray and $\gamma$-ray regimes for ISPs and HSPs. Depending on the type of blazar (and, hence, the contribution that synchrotron emission makes to the X-ray emission), leptonic models predict (a) moderate X-ray polarization, but vanishing $\gamma$-ray polarization for LSPs blazars, (b) high soft X-ray polarization, rapidly decreasing with photon energy for ISPs, and (c) high X-ray polarization and low $<200 \mathrm{MeV} \gamma$-ray polarization (though increasing towards higher energies beyond $200 \mathrm{MeV}$ ) for HSPs. 
We have outlined a method, based on optical, X-ray and $\gamma$-ray polarimetry that may allow us to confidently distinguish between leptonic and hadronic X-ray and $\gamma$-ray emission in LSP and ISP blazars. Unfortunately, given the fact that leptonic and hadronic models make identical predictions for the X-ray polarization of HBLs and $\gamma$-ray polarization in those objects is not expected to be measurable with Fermi-LAT, it is unlikely that X-ray polarimetry of HBLs will aid in distinguishing leptonic from hadronic emission. Due to the prospect of measuring the degree of order/disorder of the magnetic field in ISPs through a comparison of X-ray polarimetry with our synchrotron predictions, they might be the best candidates to identify hadronic processes. These objects will allow us to apply the method described above directly, without relying on optical/NIR measurements, and - unlike in HSPs - a measurement of the expected $\gamma$-ray polarization may be feasible using Fermi LAT or upcoming experiments.

An important aspect to point out is that high-energy polarization will not be affected by Faraday rotation due to the $\lambda^{2}$ dependence of this effect. While at radio wavelengths, Faraday rotation often substantially alters not only the orientation of the polarization direction, but may also lead to Faraday depolarization, this effect can be ignored in X-rays and $\gamma$-rays, thus revealing the true, intrinsic degree of order of the magnetic field and its directionality.

Alternative ways of distinguishing leptonic from hadronic emission scenarios for blazars rely on the potential detectability of neutrino emission expected in hadronic models (e.g., Mannheim \& Biermann 1992; Mücke \& Protheroe 2001) and characteristic variability signatures, in particular uncorrelated synchrotron and high-energy variability, which is difficult to explain in leptonic models, but may result more naturally in hadronic ones (Dimitrakoudis et al. 2012; Spanier \& Weidinger 2012). However, given the sensitivity of current and currently planned neutrino detectors, it is unclear whether neutrino signals from blazars can be detected in the forseeable future. Furthermore, also leptonic model interpretations have been suggested to explain uncorrelated synchrotron (optical - X-ray) and $\gamma$-ray variability patterns (e.g., Gutierrez et al. 2006), so that even such uncorrelated variability can not be considered a unique diagnostic for hadronic emission.

Finally, we point out that our method is not limited to blazars, but may be applied to a large variety of X-ray and $\gamma$-ray sources as long as SSC emission is produced in the Thomson regime. The high energy emission from many other X-ray and $\gamma$-ray sources (e.g., non-blazar AGN, gamma-ray bursts, microquasars, supernova remnants, ...), consists of non-thermal synchrotron and inverse Compton emission to which our existing code can be readily applied. In future work, we plan to extend our calculations to multi-zone geometries, in which anisotropic particle distributions and arbitrary magnetic-field configurations can be included. Using this code will allow us to verify the scaling arguments used to estimate 
the realistically expected degree of polarization in not perfectly homogeneous magnetic fields applied above, and to investigate the effects of varying magnetic-field geometries during $\gamma$ ray outbursts, as suggested by correlated optical $-\gamma$-ray flaring events in several blazars (e.g., Marscher et al. 2008).

\section{Acknowledgments}

We thank the referee for a helpful and constructive report. This work was funded by NASA through Fermi guest investigator grant NNX12AP20G. M.B. acknowledges support from the South African Department of Science and Technology through the National Research Foundation under NRF SARChI Chair grant No. 64789.

\section{REFERENCES}

Acciari, V. A., et al., 2009, ApJ, 707, 612

Abdo, A. A., et al., 2011, ApJ, 726, 43

Aharonian, F. A., 2000, New Astronomy, 5, 377

Aliu, E., et al., 2011, ApJ, 742, 127

Aliu, E., et al., 2012, ApJ, 750, 94

Beilicke, M. G., et al., 2012, Proc. SPIE 8507, Hard X-Ray, Gamma-Ray and Neutron Detector Physics XIV, 85071D

Böttcher, M., 2010, in proc. of "Fermi Meets Jansky", Eds. T. Savolainan, E. Ros, R. W. Porcas, \& A. Zensus; p. 41

Böttcher, M., Mause, H., \& Schlickeiser, R., 1997, A\&A, 324, 395

Böttcher, M, Reimer, A., Sweeney, K., and Prakash, A., 2013, ApJ, 768, 54

Bonometto, S., Cazzola, P., Saggion, A., 1970, A\&A, 7, 292

Bonometto, S., Saggion, A., 1973, A\&A, 23, 9

Bühler, R., et al., 2010, talk at "SCINEGHE 2010 Trieste"1]

${ }^{1}$ http://www.rolfbuehler.net/index_talks.html 
Dean, A. J., et al., 2008, Science, 321, 1183

Dimitrakoudis, S., Mastichiadis, A., Protheroe, R. J., \& Reimer, A., 2012, A\&A, 546, 120

Forot, M., Laurent, P., Grenier, I. A., Gouiffs, C., \& Lebrun, F., 2008, ApJL, 688, L29Dimitrakoudis

Ghisellini, G., Celotti, A., Fossati, G., Maraschi, L., \& Comastri, A., 1998, MNRAS, 301, 451

Gutierrez, K., et al., 2006, ApJ, 644, 742

Krawczynski, H., 2012, ApJ, 744, 30

Mannheim, K. \& Biermann, P. L., 1992, A\&A, 253, L21

Marscher, A. P., et al., 2008, Nature, 452, 966

Mücke, A., \& Protheroe, R. J., 2001, Astroparticle Physics, 15, 121

Orsi, S., 2011, Astrophys. and Space Science Transactions, 7, 43

Pearce, M., et al., 2012, pres. at IEEE Nuclear Science Symp. 2012 (arXiv:1211.5094)

Poutanen, J., 1994, ApJS, 92, 607

Rybicci, G. B., Lightman, A. P, 1985, Radiative processes in Astrophysics, Wiley-VCH

Spanier, F., \& Weidinger, M., 2012, IJMP Conf. Ser., 8, 293

Swank, J., et al., 2010, "X-Ray Polarimetry: A New Window in Astrophysics", eds. R. Bellazzini, E. Costa, G. Matt, \& G. Tagliaferri, Cambridge Univ. Press, p. 251

Tajima, H., et al., 2010, "X-Ray Polarimetry: A New Window in Astrophysics", eds. R. Bellazzini, E. Costa, G. Matt, \& G. Tagliaferri, Cambridge Univ. Press, p. 275

This preprint was prepared with the AAS LATEX macros v5.2. 